Comment. Math. Helv. 74 (1999) 548-574

(C) 1999 Birkhäuser Verlag, Basel

$0010-2571 / 99 / 040548-27 \$ 1.50+0.20 / 0$

Commentarii Mathematici Helvetici

\title{
Preprojective algebras, differential operators and a Conze embedding for deformations of Kleinian singularities
}

\author{
William Crawley-Boevey
}

\begin{abstract}
For any associative algebra $A$ over a field $K$ we define a family of algebras $\Pi^{\lambda}(A)$ for $\lambda \in K \otimes_{\mathbb{Z}} \mathrm{K}_{0}(A)$. In case $A$ is the path algebra of a quiver, one recovers the deformed preprojective algebra introduced by M. P. Holland and the author. In case $A$ is the coordinate ring of a smooth curve, the family includes the ring of differential operators for $A$ and the coordinate ring of the cotangent bundle for $\operatorname{Spec} A$. In case $A$ is quasi-free and $\Omega^{1} A$ is a finitely generated $A$-A-bimodule we prove that $\Pi^{\lambda}(A)$ is well-behaved under localization. We use this to prove a Conze embedding for deformations of Kleinian singularities.
\end{abstract}

Mathematics Subject Classification (1991). Primary 16G20; Secondary 16S32.

Keywords. Preprojective algebra, quiver, quasi-free algebra, pseudoflat epimorphism, differential operator, Kleinian singularity, Conze embedding.

If $K$ is an algebraically closed field of characteristic zero and $\Gamma$ is a non-trivial finite subgroup of $\mathrm{SL}_{2}(K)$ then the coordinate ring of the Kleinian singularity $K^{2} / \Gamma$ has a family of deformations $\mathcal{O}^{\lambda}$ where $\lambda \in Z(K \Gamma)$. They have been defined and studied in work of M. P. Holland and the author [5]. If $\lambda$ has trace zero on the regular representation of $\Gamma$, then $\mathcal{O}^{\lambda}$ is a commutative ring, and it occurs as the coordinate ring of a fibre of the semi-universal deformation of $K^{2} / \Gamma$. On the other hand, if $\lambda$ has nonzero trace on the regular representation, then $\mathcal{O}^{\lambda}$ is a non-commutative ring.

In this paper we construct an embedding $\psi_{\lambda}: \mathcal{O}^{\lambda} \rightarrow C_{\nu}$ where $\nu$ is the trace of $\lambda$ on the regular representation of $\Gamma$, and $C_{\nu}=K\langle x, y \mid x y-y x=\nu\rangle$. This is an embedding of noetherian domains, and we show that it induces an isomorphism of quotient division rings. In the commutative case $C_{\nu}$ is a polynomial ring in two variables, so the embedding is a birational map from the affine plane to a deformation of the Kleinian singularity. In the noncommutative case $C_{\nu}$ is isomorphic to the first Weyl algebra, and the embedding is reminiscent of one constructed by N. Conze [3]. We therefore call $\psi_{\lambda}$ a 'Conze embedding'.

In the work of M. P. Holland and the author, the key idea for studying deformations of Kleinian singularities was to relate them to a new class of algebras which we introduced, the 'deformed preprojective algebras' associated to quivers of extended Dynkin type. In fact, in our earliest work we constructed Conze 
embeddings. By dividing into cases according to the different types of extended Dynkin quivers, we constructed representations of the deformed preprojective algebras over $C_{\nu}$. These representations induce maps $\mathcal{O}^{\lambda} \rightarrow C_{\nu}$, and we used computer calculations to prove that these maps are injective. Instead of publishing our work, we decided to wait for a better understanding of deformed preprojective algebras, and a natural proof of the existence of Conze embeddings. This paper is the result. Although M. P. Holland is not explicitly an author of this paper, he has contributed a great deal to it.

Let $K$ be an arbitrary field. For any $K$-algebra $A$ (associative, with 1 ), and any element $\lambda \in K \otimes_{\mathbb{Z}} \mathrm{K}_{0}(A)$ we define an algebra $\Pi^{\lambda}(A)$. It is equipped with a homomorphism $A \rightarrow \Pi^{\lambda}(A)$. Note that an element $\nu \in K$ determines the element $\nu \otimes[A] \in K \otimes_{\mathbb{Z}} \mathrm{K}_{0}(A)$, and for simplicity we write $\Pi^{\nu}(A)$ rather than $\Pi^{\nu \otimes[A]}(A)$.

For finite-dimensional hereditary algebras this definition generalizes the preprojective algebras of Baer, Geigle and Lenzing [2], which we denote here by $\Pi_{\mathrm{BGL}}(A)$.

Theorem 0.1. If $A$ is finite-dimensional and hereditary then $\Pi^{0}(A) \cong \Pi_{\mathrm{BGL}}(A)$.

Our next result relates the new definition to the original deformed preprojective algebras of [5], which we denote here by $\Pi_{\mathrm{CBH}}^{\lambda}(Q)$, where $Q$ is a quiver with vertex set $I$ and $\boldsymbol{\lambda} \in K^{I}$. We identify $K^{I}$ with $K \otimes_{\mathbb{Z}} \mathrm{K}_{0}(K Q)$, with $\boldsymbol{\lambda} \in K^{I}$ corresponding to the element

$$
\lambda=\sum_{i \in I} \boldsymbol{\lambda}_{i} \otimes\left[K Q e_{i}\right] \in K \otimes_{\mathbb{Z}} \mathrm{K}_{0}(K Q)
$$

(where $e_{i}$ is the trivial path at vertex $i$ ).

Theorem 0.2. If $Q$ is a quiver and $\lambda \in K \otimes_{\mathbb{Z}} \mathrm{K}_{0}(K Q)$ then $\Pi^{\lambda}(K Q) \cong \Pi_{\mathrm{CBH}}^{\lambda}(Q)$.

Theorems 0.1 and 0.2 together imply that $\Pi_{\mathrm{BGL}}(K Q) \cong \Pi_{\mathrm{CBH}}^{0}(Q)$. This has been known for some time - it was explained to the author by C. M. Ringel - and it was used implicitly in [5]. An alternative proof has recently been written up by Ringel [13].

Theorem 0.2 includes as a special case the fact that for a polynomial ring in one variable, $\Pi^{\nu}(K[x]) \cong C_{\nu}$. It is this isomorphism which leads to the appearance of $C_{\nu}$ in the Conze embedding. When $K$ has characteristic zero, the algebra $\Pi^{1}(K[x])$ is the ring of differential operators for $K[x]$. This turns out to be no coincidence. Indeed we prove the following result.

Theorem 0.3. If $K$ is a field of characteristic zero and $A$ is the coordinate ring of a smooth affine curve over $K$, then $\Pi^{0}(A)$ is the coordinate ring of the cotangent bundle of $\operatorname{Spec} A$ and $\Pi^{1}(A)$ is the ring of differential operators for $A$.

The first three theorems deal with examples of $\Pi^{\lambda}(A)$. We now turn to the functorial properties of $\Pi^{\lambda}(A)$. In Section 5 we prove the following result. 
Theorem 0.4. Suppose that $e$ is an idempotent in an algebra $A$ with $A e A=A$. If $\lambda \in K \otimes_{\mathbb{Z}} \mathrm{K}_{0}(e A e)$, then $\Pi^{\lambda}(e A e) \cong e\left(\Pi^{\mu}(A)\right) e$, where $\mu \in K \otimes_{\mathbb{Z}} \mathrm{K}_{0}(A)$ corresponds to $\lambda$ under the natural isomorphism $\mathrm{K}_{0}(e A e) \cong \mathrm{K}_{0}(A)$.

It follows that the algebras $\Pi^{\lambda}(A)$ are well-behaved under passage to matrix rings and under Morita equivalence. For example if $A$ and $B$ are Morita-equivalent algebras, and $\lambda \in K \otimes_{\mathbb{Z}} \mathrm{K}_{0}(A)$ corresponds to $\mu \in K \otimes_{\mathbb{Z}} \mathrm{K}_{0}(B)$, under the isomorphism $\mathrm{K}_{0}(A) \cong \mathrm{K}_{0}(B)$, then $\Pi^{\lambda}(A)$ and $\Pi^{\mu}(B)$ are Morita equivalent. In the next section we show that the algebras $\Pi^{\lambda}(A)$ are also well-behaved under direct products.

According to Cuntz and Quillen [6], an algebra $A$ is quasi-free if the kernel $\Omega^{1} A$ of the multiplication map $A \otimes A \rightarrow A$ is a projective $A$ - $A$-bimodule. Such algebras are to be considered as coordinate rings of noncommutative manifolds. We say that $A$ is bimodule-finite if $\Omega^{1} A$ is a finitely generated bimodule. The following result is perhaps already known to experts.

Proposition 0.5. An algebra $A$ is bimodule-finite if and only if it has a finitely generated subalgebra $C$, such that the inclusion $C \rightarrow A$ is a ring epimorphism.

It is easy to see that path algebras are both quasi-free and bimodule-finite. Now any quasi-free algebra is hereditary, and over the complex numbers Cuntz and Quillen observed that every finite-dimensional hereditary algebra is Morita equivalent to a path algebra, so is quasi-free. In the general case, however, the situation is slightly more complicated.

Proposition 0.6. A finite-dimensional algebra $A$ is quasi-free if and only if it is hereditary and $A / \operatorname{rad} A$ is separable over $K$.

Our real reason for working in an abstract setting is in order to prove a localization theorem. For any ring homomorphism $\theta: A \rightarrow B$, the functor $B \otimes_{A}-$ defines a homomorphism $\theta_{*}: K \otimes_{\mathbb{Z}} \mathrm{K}_{0}(A) \rightarrow K \otimes_{\mathbb{Z}} \mathrm{K}_{0}(B)$. Following [1, §5], a ring epimorphism $A \rightarrow B$ is said to be pseudoflat provided that $\operatorname{Tor}_{1}^{A}(B, B)=0$. See [15, Theorem 4.8] for a number of equivalent conditions.

Theorem 0.7. If $\theta: A \rightarrow B$ is a pseudoflat epimorphism and $\lambda \in K \otimes_{\mathbb{Z}} \mathrm{K}_{0}(A)$ then there is a natural map $\Pi^{\lambda}(A) \rightarrow \Pi^{\theta_{*}(\lambda)}(B)$. If $A$ is a quasi-free bimodulefinite algebra, then so is $B$, and the diagram

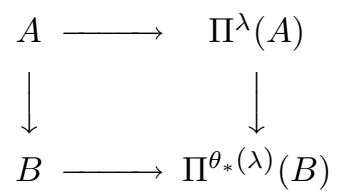

is a pushout in the category of rings. 
Now suppose that $Q$ is an extended Dynkin quiver with vertex set $I$, and for simplicity suppose that $K$ is an algebraically closed field. Let $\delta \in \mathbb{Z}^{I}$ be the minimal positive imaginary root for $Q$. By using universal localization one can easily construct a pseudoflat epimorphism $\theta: A \rightarrow \mathrm{M}_{N}(K[x])$ such that the general representation of $K Q$ of dimension vector $\delta$ is the restriction of a $\mathrm{M}_{N}(K[x])$ module. (It follows that $N=\sum_{i} \delta_{i}$.) If $\lambda \in K \otimes_{\mathbb{Z}} \mathrm{K}_{0}(K Q)$ ), by the theorem there is an induced a pseudoflat epimorphism $\theta_{\lambda}$ from $\Pi^{\lambda}(K Q)$ to

$$
\Pi^{\theta_{*}(\lambda)}\left(\mathrm{M}_{N}(K[x])\right) \cong \mathrm{M}_{N}\left(\Pi^{\nu}(K[x])\right) \cong \mathrm{M}_{N}\left(C_{\nu}\right)
$$

where in fact $\nu=\sum_{i} \boldsymbol{\lambda}_{i} \delta_{i}$. By using the representation theory of $Q$ we prove the following result.

Theorem 0.8. If $Q$ is an extended Dynkin quiver, $K$ is an algebraically closed field and $\lambda \in K \otimes_{\mathbb{Z}} \mathrm{K}_{0}(K Q)$ then $\Pi^{\lambda}(K Q)$ is a prime noetherian ring of GelfandKirillov dimension 2. Moreover $\theta_{\lambda}: \Pi^{\lambda}(K Q) \rightarrow \mathrm{M}_{N}\left(C_{\nu}\right)$ is injective, and it induces an isomorphism on simple artinian quotient rings.

In an appendix we use the methods of this paper to study the variety of representations of $\Pi^{0}(K Q)$ of dimension $\delta$.

We finally return to Kleinian singularities. Assume that $K$ is algebraically closed of characteristic zero, and let $\Gamma$ be a finite subgroup of $\mathrm{SL}_{2}(K)$. Let $Q$ be an orientation of the McKay quiver of $\Gamma$, and let 0 be an extending vertex. Recall that there is an isomorphism $\mathcal{O}^{\lambda} \cong e_{0} \Pi^{\lambda}(K Q) e_{0}$, where $\lambda \in Z(K \Gamma)$ is identified with $\lambda \in K^{I}$ by letting $\lambda_{i}$ be the trace of $\lambda$ on the $i$ th irreducible representation of $\Gamma$. Here is the result mentioned at the start of the introduction.

Theorem 0.9. There is an embedding $\psi_{\lambda}: \mathcal{O}^{\lambda} \rightarrow C_{\nu}$ where $\nu$ is the trace of $\lambda$ on the regular representation of $\Gamma$. Moreover $\psi_{\lambda}$ induces an isomorphism on quotient division rings.

\section{Definition of $\Pi^{\lambda}(A)$}

Let $A$ be an algebra (associative, with 1 ) over a field $K$. Recall that $A$ - $A$-bimodules are the same as $A^{e}$-modules, where $A^{e}=A \otimes A^{o p}$. (Unadorned tensor products are always over the field $K$.) The universal derivation bimodule, or bimodule of noncommutative differential 1-forms is the kernel $\Omega^{1} A$ of the multiplication map $A \otimes A \rightarrow A$. See for example [1] or [6].

If $M$ is an $A$ - $A$-bimodule, we write $\operatorname{Der}(A, M)$ for the space of derivations from $A$ to $M$. It is isomorphic to $\operatorname{Hom}_{A^{e}}\left(\Omega^{1} A, M\right)$, a homomorphism $\theta$ giving rise to the derivation $d$ with $d(a)=\theta(a \otimes 1-1 \otimes a)$.

The space $\operatorname{Der}(A, A \otimes A)$ becomes an $A$-A-bimodule via $a d b=\left(r_{b} \otimes \ell_{a}\right) d$ where $\ell_{a}, r_{b}: A \rightarrow A$ denote left multiplication by $a$ and right multiplication 
by $b$ respectively. We write $\Delta$ (or $\Delta_{A}$ ) for the derivation $A \rightarrow A \otimes_{K} A$ with $\Delta(a)=a \otimes 1-1 \otimes a$.

If $M$ is an $A$ - $A$-bimodule we write $\mathrm{T}_{A} M$ for the tensor algebra of $M$ over $A$. For any $a \in A$ we define $\Pi^{a}(A)=\mathrm{T}_{A} \operatorname{Der}(A, A \otimes A) /(\Delta-a)$. We consider it as an $A$-ring, that is, as an algebra equipped with homomorphism $A \rightarrow \Pi^{a}(A)$. We have the following elementary results.

Lemma 1.1. If $u$ is a unit in the centre of $A$ and $a \in A$, then $\Pi^{a}(A) \cong \Pi^{u a}(A)$.

Proof. Multiplication on the left by $u^{-1}$ induces an automorphism of the bimodule $\operatorname{Der}(A, A \otimes A)$, and hence there is an algebra automorphism of $\mathrm{T}_{A} \operatorname{Der}(A, A \otimes A)$ which is the identity on $A$, but sends $\Delta$ to $u^{-1} \Delta$. Under this automorphism the ideal $(\Delta-a)$ is sent to $\left(u^{-1} \Delta-a\right)=(\Delta-u a)$.

Lemma 1.2. Up to isomorphism $\Pi^{a}(A)$ depends only on the image of a in $\mathrm{H}_{0}(A)=$ $A /[A, A]$.

Proof. Write $M=\operatorname{Der}(A, A \otimes A)$. Given $b, c \in A$, it suffices to construct an automorphism of $\mathrm{T}_{A} M$ sending $\Delta-a$ to $\Delta-a+[b, c]$. By the universal property of tensor algebras, the homomorphisms $\theta: \mathrm{T}_{A} M \rightarrow \mathrm{T}_{A} M$ which are the identity on $A$ are in 1-1 correspondence with $A$ - $A$-bimodule maps $M \rightarrow \mathrm{T}_{A} M$. Taking the bimodule map sending $d$ to $d+\sum_{i} y_{i} c x_{i}$, where $x_{i}$ and $y_{i}$ are defined by $d(b)=$ $\sum_{i} x_{i} \otimes y_{i}$, it is clear that the resulting homomorphism $\theta$ is an automorphism, and that $\theta(\Delta-a)=\Delta-a+[b, c]$, as required.

Let $\operatorname{tr}: \mathrm{K}_{0}(A) \rightarrow \mathrm{H}_{0}(A)=A /[A, A]$ be the trace map, sending the class of a projective module $P$ to the image in $A /[A, A]$ of the trace of any idempotent $e \in \mathrm{M}_{n}(A)$ with image isomorphic to $P$. This map extends to a linear map $K \otimes_{\mathbb{Z}} \mathrm{K}_{0}(A) \rightarrow \mathrm{H}_{0}(A)$ also denoted tr.

Definition 1.3. If $\lambda \in K \otimes_{\mathbb{Z}} \mathrm{K}_{0}(A)$ then

$$
\Pi^{\lambda}(A)=\Pi^{a_{\lambda}}(A)=\mathrm{T}_{A} \operatorname{Der}(A, A \otimes A) /\left(\Delta-a_{\lambda}\right)
$$

where $a_{\lambda}$ is any lift of $\operatorname{tr}(\lambda)$ to $A$.

The previous lemma shows that $\Pi^{\lambda}(A)$ does not depend on the choice of $a_{\lambda}$. If $a, b \in A$ then $a \Delta b$ is the derivation with $(a \Delta b)(x)=x b \otimes a-b \otimes a x$ for $x \in A$. Thus the $A$ - $A$-sub-bimodule of $\operatorname{Der}(A, A \otimes A)$ generated by $\Delta$ is the set of inner derivations. Therefore $\operatorname{Der}(A, A \otimes A) / A \Delta A \cong \mathrm{H}^{1}(A, A \otimes A)$, which has the following consequence.

Lemma 1.4. $\Pi^{0} A \cong \mathrm{T}_{A} \mathrm{H}^{1}(A, A \otimes A)$.

Any tensor algebra $\mathrm{T}_{A} M$ is naturally graded, with $\left(\mathrm{T}_{A} M\right)_{n}$ being the $n$th 
tensor power of $M$. Thus the lemma gives a grading of $\Pi^{0}(A)$. On the other hand the algebra $\Pi^{a}(A)$ is filtered

$$
\Pi^{a}(A)_{\leq 0} \subseteq \Pi^{a}(A)_{\leq 1} \subseteq \Pi^{a}(A)_{\leq 2} \subseteq \ldots,
$$

where $\Pi^{a}(A)_{\leq n}$ is the image in $\Pi^{a}(A)$ of $\sum_{i=0}^{n}\left(\mathrm{~T}_{A} \operatorname{Der}(A, A \otimes A)\right)_{i}$. As usual one can consider the associated graded algebra $\operatorname{gr} \Pi^{a}(A)$, and there is the following result.

Lemma 1.5. There is a natural surjective graded algebra map $\Pi^{0}(A) \rightarrow \operatorname{gr} \Pi^{a}(A)$.

Proof. An algebra homomorphism $\phi: \Pi^{0}(A) \rightarrow \operatorname{gr} \Pi^{a}(A)$ is determined by an algebra homomorphism $A \rightarrow \Pi^{a}(A)_{\leq 0}$, which we take to be the natural map, and an $A$ - $A$-bimodule map $g: \mathrm{H}^{1}(A, A \otimes A) \rightarrow\left(\operatorname{gr} \Pi^{a}(A)\right)_{1}$. For $\phi$ to be surjective, we need $g$ to be surjective, so it suffices to show that $\left(\operatorname{gr} \Pi^{a}(A)\right)_{1}$ is naturally a quotient of $\mathrm{H}^{1}(A, A \otimes A)$. Now by definition $\left(\operatorname{gr} \Pi^{a}(A)\right)_{1}=(A \oplus \operatorname{Der}(A, A \otimes A)) / S$, where $S=A+I \cap(A \oplus \operatorname{Der}(A, A \otimes A))$ and $I$ is the ideal $(\Delta-a)$ in $\mathrm{T}_{A} \operatorname{Der}(A, A \otimes A)$. The result follows since $S$ contains $A \oplus A \Delta A$.

\section{Finite-dimensional hereditary algebras}

Let $D$ be the duality $\operatorname{Hom}_{K}(-, K)$.

Lemma 2.1. For any algebra $A$ there is a natural isomorphism $\operatorname{Ext}_{A}^{1}(D Y, X) \cong$ $\mathrm{H}^{1}(A, X \otimes Y)$ for $X$ and $Y$ left $A$-modules, with $Y$ finite-dimensional.

Proof. Tensoring the defining sequence for $\Omega^{1} A$ with $D Y$ one obtains an exact sequence

$$
0 \rightarrow \Omega^{1} A \otimes_{A} D Y \rightarrow A \otimes D Y \rightarrow D Y \rightarrow 0 .
$$

Since $A \otimes D Y$ is projective, if $X$ is a left $A$-module one obtains

$$
\cdots \rightarrow \operatorname{Hom}_{A}(A \otimes D Y, X) \rightarrow \operatorname{Hom}_{A}\left(\Omega^{1} A \otimes_{A} D Y, X\right) \rightarrow \underset{A}{\operatorname{Ext}}(D Y, X) \rightarrow 0 .
$$

Now $\operatorname{Hom}_{A}\left(\Omega^{1} A \otimes_{A} D Y, X\right)$ can be identified with $\operatorname{Hom}_{A^{e}}\left(\Omega^{1} A, X \otimes Y\right)$, so with $\operatorname{Der}(A, X \otimes Y)$. Also we can identify $\operatorname{Hom}_{A}(A \otimes D Y, X)$ with $Y \otimes X$, and then the map $Y \otimes X \rightarrow \operatorname{Der}(A, X \otimes Y)$ sends an element of $Y \otimes X$ to the corresponding inner derivation.

Lemma 2.2. If $A$ is a finite-dimensional hereditary algebra then there is a natural isomorphism $\operatorname{Ext}^{1}(D A, X) \cong \mathrm{H}^{1}(A, A \otimes A) \otimes_{A} X$ for $X$ a left $A$-module. 
Proof. Since $A$ is hereditary the functor $\operatorname{Ext}^{1}(D A,-)$ is right exact. Since it also commutes with direct limits it is naturally isomorphic to the tensor product functor $\operatorname{Ext}^{1}(D A, A) \otimes_{A}-$.

If $A$ is a finite-dimensional hereditary algebra then the inverse AuslanderReiten translation is the functor $\tau^{-}$defined by $\tau^{-}(X)=\operatorname{Ext}^{1}(D A, X)$. The algebra $\Pi_{\mathrm{BGL}}(A)$ of $[2, \S 3]$ is

$$
\bigoplus_{n=0}^{\infty} \operatorname{Hom}_{A}\left(A, \tau^{-n}(A)\right),
$$

with the product defined by $u v=\tau^{-m}(u) \circ v$ for elements $u \in \operatorname{Hom}_{A}\left(A, \tau^{-n}(A)\right)$ and $v \in \operatorname{Hom}_{A}\left(A, \tau^{-m}(A)\right)$.

Theorem 2.3. If $A$ is finite-dimensional and hereditary then $\Pi^{0}(A) \cong \Pi_{\mathrm{BGL}}(A)$.

Proof. Combine Lemmas 1.4 and 2.2 with [2, Proposition 3.1].

\section{Deformed preprojective algebras}

Let $Q$ be a quiver with vertex set $I$ and let $K Q$ be its path algebra. Let $e_{i} \in K Q$ be the trivial path at vertex $i$. Recall that $K^{I}$ is identified with $K \otimes_{\mathbb{Z}} \mathrm{K}_{0}(K Q)$, with $\boldsymbol{\lambda} \in K^{I}$ corresponding to the element

$$
\lambda=\sum_{i \in I} \boldsymbol{\lambda}_{i} \otimes\left[K Q e_{i}\right] \in K \otimes_{\mathbb{Z}} \mathrm{K}_{0}(K Q)
$$

Let $\bar{Q}$ be the quiver obtained from $Q$ by adjoining an arrow $a^{*}: j \rightarrow i$ for each arrow $a: i \rightarrow j$ in $Q$. The next result shows that $\Pi^{\lambda}(K Q)$ coincides with the deformed preprojective algebra $\Pi_{\mathrm{CBH}}^{\boldsymbol{\lambda}}(Q)$ of $[5]$.

Theorem 3.1. If $\lambda \in K \otimes_{\mathbb{Z}} \mathrm{K}_{0}(K Q)$ then $\Pi^{\lambda}(K Q) \cong K \bar{Q} / J$ where $J$ is the ideal generated by $\sum_{a \in Q}\left[a, a^{*}\right]-\sum_{i \in I} \boldsymbol{\lambda}_{i} e_{i}$.

Proof. There is a standard projective resolution of $K Q$ as a $K Q$ - $K Q$-bimodule

$$
0 \rightarrow \bigoplus_{a: i \rightarrow j \text { in } Q} K Q e_{j} \otimes e_{i} K Q \stackrel{f}{\rightarrow} \bigoplus_{i \in I} K Q e_{i} \otimes e_{i} K Q \stackrel{\text { mult }}{\rightarrow} K Q \rightarrow 0
$$

where $f$ sends $\left(e_{j} \otimes e_{i}\right)_{a}$ to $\left(e_{j} \otimes a\right)_{j}-\left(a \otimes e_{i}\right)_{i}$. Identifying

$$
K Q \otimes K Q=\bigoplus_{r, s \in I} K Q e_{r} \otimes e_{s} K Q,
$$


one obtains an exact sequence

$$
0 \rightarrow \bigoplus_{a: i \rightarrow j} K Q e_{j} \otimes e_{i} K Q \oplus \bigoplus_{r \neq s} K Q e_{r} \otimes e_{s} K Q \stackrel{g}{\rightarrow} K Q \otimes K Q \stackrel{\text { mult }}{\rightarrow} K Q \rightarrow 0
$$

where $g$ sends $\left(e_{j} \otimes e_{i}\right)_{a}$ to $e_{j} \otimes a-a \otimes e_{i}$ and $\left(e_{r} \otimes e_{s}\right)_{r s}$ to $e_{r} \otimes e_{s}$.

If $M$ is a $K Q-K Q$-bimodule, then $\operatorname{Hom}_{K Q^{e}}\left(K Q e_{i} \otimes e_{j} K Q, M\right) \cong e_{i} M e_{j}$, so if $M=K Q \otimes K Q$ the Hom space is isomorphic to $e_{i} K Q \otimes K Q e_{j}$. Now this tensor product is over $K$, so one can swap the order of the terms and write $K Q e_{j} \otimes e_{i} K Q$. By doing this, one clearly sees the $K Q-K Q$-bimodule structure of this space.

Now the left hand term of the exact sequence $(\dagger)$ is $\Omega^{1} K Q$, and computing its homomorphisms to $K Q \otimes K Q$ one obtains

$$
\operatorname{Der}(K Q, K Q \otimes K Q) \cong \bigoplus_{a: i \rightarrow j} K Q e_{i} \otimes e_{j} K Q \oplus \bigoplus_{r \neq s} K Q e_{s} \otimes e_{r} K Q
$$

Thus $\mathrm{T}_{K Q} \operatorname{Der}(K Q, K Q \otimes K Q)$ is identified with the path algebra of the quiver $\tilde{Q}$ obtained from $\bar{Q}$ by adding an arrow $c_{r s}: r \rightarrow s$ for each pair of vertices $r \neq s$. Also $\Delta$ corresponds to the map $g$, so to the element

$$
\sum_{a: i \rightarrow j}\left(a \otimes e_{j}-e_{i} \otimes a\right)_{a}+\sum_{r \neq s}\left(e_{s} \otimes e_{r}\right)_{r s}
$$

Now with the identification of $K^{I}$ and $K \otimes_{\mathbb{Z}} \mathrm{K}_{0}(K Q)$, the element $\sum_{i} \boldsymbol{\lambda}_{i} e_{i}$ is a lift to $K Q$ of $\operatorname{tr}(\lambda) \in K Q /[K Q, K Q]$. Thus $\Pi^{\lambda}(K Q) \cong K \tilde{Q} /(\xi)$ where

$$
\xi=\sum_{a: i \rightarrow j}\left(a a^{*}-a^{*} a\right)+\sum_{r \neq s} c_{r s}-\sum_{i} \boldsymbol{\lambda}_{i} e_{i}
$$

Clearly the ideal generated by $\xi$ is also generated by the elements $\sum_{i} e_{i} \xi e_{i}$ with $i \in I$ and $e_{s} \xi e_{r}$ with $r \neq s$. These are $\sum_{a \in Q}\left[a, a^{*}\right]-\sum_{i=1}^{n} \lambda_{i} e_{i}$ and $c_{r s}$ respectively. The result follows.

The path algebra of the quiver with one vertex and one loop is a polynomial ring $K[x]$, so we have the following special case.

Corollary 3.2. If $\nu \in K$ then $\Pi^{\nu}(K[x]) \cong K\langle x, y \mid x y-y x=\nu\rangle$.

\section{Differential operators on curves}

Throughout this section $A$ is the coordinate ring of a smooth affine curve. Thus it is a commutative domain, finitely generated over $K$, of Krull dimension 1 , and it is smooth over $K$, so for any commutative $K$-algebra $C$ and any ideal $I$ in 
$C$ with $I^{2}=0$, any $K$-algebra homomorphism $A \rightarrow C / I$ lifts to a $K$-algebra homomorphism $A \rightarrow C$. A suitable reference for smooth algebras is $[10, \S \S 25-$ 30]. The following result is due to Schelter [14] (at least when $K$ is algebraically closed).

Lemma 4.1. $\Omega^{1} A$ is a rank 1 projective $A^{e}$-module. Thus $A$ is quasi-free.

Proof. Since $A$ is smooth over $K$, so is $A^{e}$, so for any maximal ideal $\mathfrak{m}$ of $A^{e}$ the localization $\left(A^{e}\right)_{\mathfrak{m}}$ is regular of dimension 2 . Localizing the defining sequence for $\Omega^{1} A$ at $\mathfrak{m}$ gives an exact sequence

$$
0 \rightarrow\left(\Omega^{1} A\right)_{\mathfrak{m}} \rightarrow\left(A^{e}\right)_{\mathfrak{m}} \rightarrow A \otimes_{A^{e}}\left(A^{e}\right)_{\mathfrak{m}} \rightarrow 0
$$

and it suffices to prove that the ideal $\left(\Omega^{1} A\right)_{\mathfrak{m}}$ of $\left(A^{e}\right)_{\mathfrak{m}}$ is projective. This is certainly true if $\mathfrak{m}$ doesn't contain $\Omega^{1} A$, for then some element of $\Omega^{1} A$ is invertible in $\left(A^{e}\right)_{\mathfrak{m}}$, so $\left(\Omega^{1} A\right)_{\mathfrak{m}}=\left(A^{e}\right)_{\mathfrak{m}}$. Thus suppose that $\mathfrak{m}$ contains $\Omega^{1} A$, so $\mathfrak{m}$ is the inverse image under the multiplication map $A^{e} \rightarrow A$ of a maximal ideal $\mathfrak{n}$ of $A$. This implies that $A \otimes_{A^{e}}\left(A^{e}\right)_{\mathfrak{m}} \cong A_{\mathfrak{n}}$. Now if $a$ is a nonzero element of $\mathfrak{n}$ then $1 \otimes a \in \mathfrak{m}$ and since $1 \otimes a$ acts as a non zero-divisor on $A_{\mathfrak{n}}$, the $\left(A^{e}\right)_{\mathfrak{m}}$-module $A_{\mathfrak{n}}$ has depth at least 1 . It follows that it has projective dimension at most 1 , so that $\left(\Omega^{1} A\right)_{\mathfrak{m}}$ is projective.

Since $A^{e}$ is commutative, any Hom space between $A$ - $A$-bimodules is naturally an $A$ - $A$-bimodule. In particular $\operatorname{Der}(A, M)=\operatorname{Hom}_{A^{e}}\left(\Omega^{1} A, M\right)$ is an $A$ - $A$ bimodule for any $M$.

Lemma 4.2. $\operatorname{Der}\left(A, \Omega^{1} A\right)$ is a free $A$-A-bimodule generated by $\Delta$.

Proof. It is the endomorphism ring of a rank 1 projective $A^{e}$-module.

Applying $\operatorname{Hom}_{A^{e}}\left(\Omega^{1} A,-\right)$ to the defining sequence for $\Omega^{1} A$, we obtain a sequence

$$
0 \rightarrow \operatorname{Der}\left(A, \Omega^{1} A\right) \rightarrow \operatorname{Der}(A, A \otimes A) \rightarrow \operatorname{Der}(A, A) \rightarrow 0
$$

so by the previous lemma $\mathrm{H}^{1}(A, A \otimes A)$ is naturally isomorphic to $\operatorname{Der}(A, A)$. Now $\operatorname{Der}(A, A)$ is a rank 1 projective $A$-module, so its tensor algebra over $A$ coincides with its symmetric algebra, and we have the following result.

Theorem 4.3. $\Pi^{0}(A)$ is isomorphic to the coordinate ring $K\left[\mathrm{~T}^{*} \operatorname{Spec} A\right]$ of the cotangent bundle of $\operatorname{Spec} A$.

We now investigate the relationship between $\Pi^{1}(A)$ and differential operators. Let $D(A)$ be the ring of differential operators for $A$. Elements of $A$ are identified with the differential operators of order 0 , with $a \in A$ corresponding to the homothety $a \operatorname{Id}_{A}$. We write $D_{\leq 1}(A)$ for the set of differential operators of order $\leq 1$. It 
becomes an $A$ - $A$-bimodule by composition of maps, and there is an exact sequence of $A$ - $A$-bimodules

$$
0 \longrightarrow A \longrightarrow D_{\leq 1}(A) \stackrel{g}{\longrightarrow} \operatorname{Der}(A, A) \longrightarrow 0
$$

where $g$ sends a differential operator $d$ to $d-d(1) \operatorname{Id}_{A}$. Note that $\operatorname{Der}(A, A)$ is a subspace of $D_{\leq 1}(A)$ complementary to $A \operatorname{Id}_{A}$, but it is not a sub-bimodule.

Lemma 4.4. The evaluation map $A \otimes \operatorname{Der}(A, A) \rightarrow A$ is surjective.

Proof. If $a \in A$ and $h \in \operatorname{Hom}_{A^{e}}\left(\Omega^{1} A, A\right)$ then since $A$ is commutative, the assignment $d(x)=h(a x \otimes 1-a \otimes x)$ defines a derivation $A \rightarrow A$. Now the assertion follows since firstly $\Omega^{1} A$ is generated as a left $A$-module by elements of the form $x \otimes 1-1 \otimes x$, and secondly the natural map

$$
\Omega^{1} A \otimes_{A^{e}} \operatorname{Hom}_{A^{e}}\left(\Omega^{1} A, A\right) \rightarrow A
$$

is an isomorphism (since $\Omega^{1} A$ is a rank 1 projective $A^{e}$-module).

Lemma 4.5. $A \operatorname{Id}_{A}$ is a superfluous $A$-A-sub-bimodule of $D_{\leq 1}(A)$. That is, if $M$ is an $A$-A-sub-bimodule of $D_{\leq 1}(A)$ and $M+A \operatorname{Id}_{A}=D_{\leq 1}(A)$, then $M=D_{\leq 1}(A)$.

Proof. By assumption any derivation $d \in \operatorname{Der}(A, A)$ can be written as the sum of an element $m \in M$ and a homothety $a \operatorname{Id}_{A}$. Now if $x \in A$ then $d(x) y=d(x y)-x d(y)$ for $y \in A$, so

$$
d(x) \operatorname{Id}_{A}=d \circ\left(x \operatorname{Id}_{A}\right)-\left(x \operatorname{Id}_{A}\right) \circ d=m \circ\left(x \operatorname{Id}_{A}\right)-\left(x \operatorname{Id}_{A}\right) \circ m \in M .
$$

Thus $A \operatorname{Id}_{A} \subseteq M$ by the previous lemma.

Lemma 4.6. There are surjective bimodule maps $\theta, \phi$ giving rise to a commutative diagram with exact rows

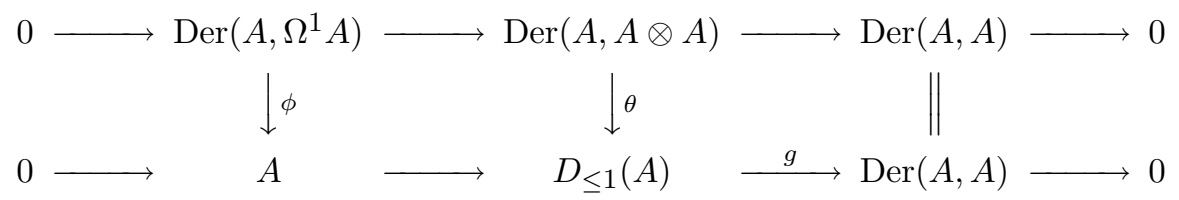

Proof. Since $\operatorname{Der}(A, A \otimes A)$ is a projective bimodule, the map from $\operatorname{Der}(A, A \otimes A)$ to $\operatorname{Der}(A, A)$ lifts to a map $\theta$. Now $\theta$ is surjective since $A \operatorname{Id}_{A}$ is superfluous in $D_{\leq 1}(A)$. The result follows.

Theorem 4.7. If $K$ has characteristic zero and $A$ is the coordinate ring of a smooth affine curve, then $\Pi^{1}(A)$ is isomorphic to the ring $D(A)$ of differential operators for $A$. 
Proof. Since the map $\phi$ in Lemma 4.6 is surjective and $\operatorname{Der}\left(A, \Omega^{1} A\right)$ is generated by $\Delta$, the element $u=\phi(\Delta)$ must be a unit in $A$. Now $\theta$ induces a homomorphism $\psi: \Pi^{u}(A) \rightarrow D(A)$ of filtered rings, and there is a commutative square

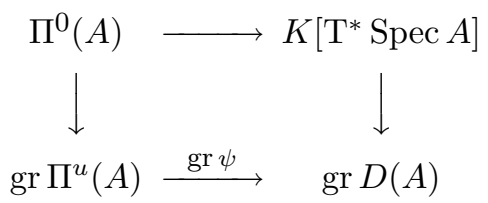

where the top map is the isomorphism of Theorem 4.3, the left hand vertical map is as in Lemma 1.5, and the right hand vertical map is the natural isomorphism resulting from the fact that $A$ is smooth and $K$ has characteristic zero. It follows that $\operatorname{gr} \psi$ is an isomorphism, and then the result follows since $\Pi^{1}(A) \cong \Pi^{u}(A)$ by Lemma 1.1.

\section{Morita equivalence}

In this section $A$ is an algebra and $e \in A$ is an idempotent with $A e A=A$, so that $A$ is Morita equivalent to $e A e$. We define $f=1-e$.

Lemma 5.1. If $M$ is an $A$-A-bimodule, then the assignment sending $d$ to the map $a \mapsto e d(a) e$ induces a surjective linear map $\operatorname{Der}(A, M) \rightarrow \operatorname{Der}(e A e, e M e)$ whose kernel is the set of inner derivations $a \mapsto a m-m a$ with $m \in f M e \oplus e M f \oplus f M f$.

Proof. Let $X=(A f \otimes e A) \oplus(A e \otimes f A) \oplus(A f \otimes f A)$. Since $A e A=A$, it follows that $A \otimes A=\Omega^{1} A+A e \otimes e A$. Thus the projection $A \otimes A \rightarrow X$ induces an exact sequence

$$
0 \rightarrow \Omega^{1} A \cap A e \otimes e A \rightarrow \Omega^{1} A \rightarrow X \rightarrow 0 .
$$

This is split exact since $X$ is a projective bimodule, so it induces an exact sequence

$$
0 \rightarrow \operatorname{Hom}_{A^{e}}(X, M) \stackrel{\theta}{\rightarrow} \operatorname{Hom}_{A^{e}}\left(\Omega^{1} A, M\right) \stackrel{\phi}{\rightarrow} \operatorname{Hom}_{A^{e}}\left(\Omega^{1}(A) \cap A e \otimes e A, M\right) \rightarrow 0 .
$$

Now $e\left(\Omega^{1}(A) \cap A e \otimes e A\right) e=\Omega^{1}(e A e)$, so by Morita equivalence the last term can be identified with the space of $e A e$-eAe-bimodule homomorphisms from $\Omega^{1}(e A e)$ to $e M e$, and hence with $\operatorname{Der}(e A e, e M e)$. Identifying also the middle term with $\operatorname{Der}(A, M)$, the map $\phi$ is as stated. Now the left hand term is isomorphic to $f M e \oplus e M f \oplus f M f$, and the map $\theta$ sends an element $m$ of this direct sum to the inner derivation $a \mapsto a m-m a$, as required.

Lemma 5.2. If $J$ is the $A$-A-sub-bimodule of $\operatorname{Der}(A, A \otimes A)$ generated by e $\Delta f$, $f \Delta e$ and $f \Delta f$, and $L=\operatorname{Der}(A, A \otimes A) / J$, then there is an isomorphism of eAeeAe-bimodules

$$
e L e \rightarrow \operatorname{Der}(e A e, e A e \otimes e A e)
$$


sending $e \bar{\Delta} e$ to $\Delta_{e A e}$.

Proof. One can identify $e \operatorname{Der}(A, A \otimes A) e$ with $\operatorname{Der}(A, A e \otimes e A)$, and then the lemma follows from the previous one.

There are inverse isomorphisms between $\mathrm{K}_{0}(e A e)$ and $\mathrm{K}_{0}(A)$ induced by the functors $P \mapsto A e \otimes_{e A e} P$ and $Q \mapsto e Q$ on projective modules. This enables one to identify $K \otimes_{\mathbb{Z}} \mathrm{K}_{0}(e A e)$ and $K \otimes_{\mathbb{Z}} \mathrm{K}_{0}(A)$.

Theorem 5.3. If $\lambda \in K \otimes_{\mathbb{Z}} \mathrm{K}_{0}(e A e)$ then $\Pi^{\lambda}(e A e) \cong e\left(\Pi^{\mu}(A)\right)$, where $\mu$ is the corresponding element of $K \otimes_{\mathbb{Z}} \mathrm{K}_{0}(A)$.

Proof. It suffices to show that $\Pi^{a}(e A e) \cong e\left(\Pi^{a} A\right) e$ for $a \in e A e$, as there is a commutative square

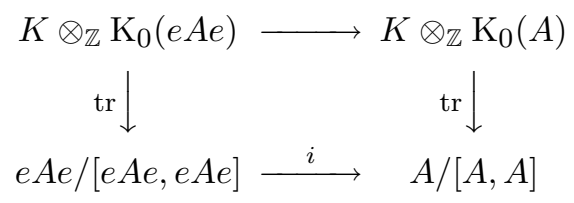

where $i$ is induced by the inclusion of $e A e$ in $A$, and the top map is the bijection mentioned above. Now

$$
\begin{aligned}
\Pi^{a}(A) & =\left(\mathrm{T}_{A} \operatorname{Der}(A, A \otimes A)\right) /(\Delta-a) \\
& =\left(\mathrm{T}_{A} \operatorname{Der}(A, A \otimes A)\right) /(e \Delta f, f \Delta e, f \Delta f, e \Delta e-a) \\
& \cong \mathrm{T}_{A} L /(e \bar{\Delta} e-a)
\end{aligned}
$$

where $L$ is as in the previous lemma. Thus

$$
\begin{aligned}
e \Pi^{a}(A) e & \cong e\left[\mathrm{~T}_{A} L /(e \bar{\Delta} e-a)\right] e \cong e\left(\mathrm{~T}_{A} L\right) e /(e \bar{\Delta} e-a) \\
& \cong \mathrm{T}_{e A e}(e L e) /(e \bar{\Delta} e-a) \cong \Pi^{a}(e A e)
\end{aligned}
$$

by the previous lemma.

If $A$ and $B$ are Morita-equivalent algebras, then there is an equivalence from the category of left $A$-modules to the category of left $B$-modules. This induces a bijection from $K \otimes_{\mathbb{Z}} \mathrm{K}_{0}(A)$ to $K \otimes_{\mathbb{Z}} \mathrm{K}_{0}(B)$. In particular this applies to the algebras $A$ and $\mathrm{M}_{n}(A)$.

Corollary 5.4. If $\lambda \in K \otimes_{\mathbb{Z}} \mathrm{K}_{0}\left(\mathrm{M}_{n}(A)\right)$ then $\Pi^{\lambda}\left(\mathrm{M}_{n}(A)\right) \cong \mathrm{M}_{n}\left(\Pi^{\mu}(A)\right)$ where $\mu$ is the corresponding element of $K \otimes_{\mathbb{Z}} \mathrm{K}_{0}(A)$.

Proof. The matrix units $e_{i j}(1 \leq i, j \leq n)$ for $\mathrm{M}_{n}(A)$ induce matrix units in $\Pi^{\lambda}\left(\mathrm{M}_{n}(A)\right)$. Thus $\Pi^{\lambda}\left(\mathrm{M}_{n}(A)\right) \cong \mathrm{M}_{n}(R)$ where $R=e_{11} \Pi^{\lambda}\left(\mathrm{M}_{n}(A)\right) e_{11}$, and then $R \cong \Pi^{\mu}(A)$ by the theorem. 
Corollary 5.5. If $A$ and $B$ are Morita-equivalent algebras, and $\lambda \in K \otimes_{\mathbb{Z}} \mathrm{K}_{0}(A)$ corresponds to $\mu \in K \otimes_{\mathbb{Z}} \mathrm{K}_{0}(B)$, then $\Pi^{\lambda}(A)$ and $\Pi^{\mu}(B)$ are Morita equivalent.

Proof. It suffices to prove this in two cases, when $B=\mathrm{M}_{n}(A)$, and when $B=e A e$ with $e$ an idempotent with $A e A=A$; see for example [11, Proposition 3.5.6]. The first corollary deals with the case of a matrix ring. The theorem deals with the other case, for $e$ is an idempotent in $\Pi^{\lambda}(A)$ with $\Pi^{\lambda}(A) e \Pi^{\lambda}(A)=\Pi^{\lambda}(A)$, so that $\Pi^{\lambda}(A)$ is Morita equivalent to $e \Pi^{\lambda}(A) e \cong \Pi^{\mu}(e A e)$.

\section{Products}

In this section we suppose that $A$ decomposes as a direct product of two algebras, $A=B \times C$. We identify $B$ and $C$ as subsets of $A$ with $A=B \oplus C$ and $B C=C B=$ 0 . This leads to a decomposition $1=e+f$ with $e \in B$ and $f \in C$ idempotents. There is a natural isomorphism $\mathrm{K}_{0}(A) \cong \mathrm{K}_{0}(B) \times \mathrm{K}_{0}(C)$, and if $\lambda \in K \otimes_{\mathbb{Z}} \mathrm{K}_{0}(B)$ and $\mu \in K \otimes_{\mathbb{Z}} \mathrm{K}_{0}(C)$ we write $(\lambda, \mu)$ for the corresponding element of $K \otimes_{\mathbb{Z}} \mathrm{K}_{0}(A)$. We prove that $\Pi^{(\lambda, \mu)}(A) \cong \Pi^{\lambda}(B) \times \Pi^{\mu}(C)$.

Lemma 6.1. If I is the $A$-A-sub-bimodule of $\operatorname{Der}(A, A \otimes A)$ generated by $e \Delta f$ and $f \Delta e$ then there is an isomorphism

$$
\operatorname{Der}(A, A \otimes A) / I \cong \operatorname{Der}(B, B \otimes B) \oplus \operatorname{Der}(C, C \otimes C)
$$

with $\overline{e \Delta_{A} e}$ corresponding to $\Delta_{B}$ and $\overline{f \Delta_{A} f}$ corresponding to $\Delta_{C}$

Proof. Clearly $\operatorname{Der}(B, B \otimes B) \oplus \operatorname{Der}(C, C \otimes C)$ can be identified with

$$
D=\{d \in \operatorname{Der}(A, A \otimes A) \mid d(e)=0\},
$$

so it suffices to prove that $D$ and $I$ are complementary. Now $I$ consists of all inner derivations of the form $s(a)=a(p+q)-(p+q) a$ with $p \in e A \otimes A f$ and $q \in f A \otimes A e$, and since $s(e)=q-p$, it follows that $D \cap I=0$. On the other hand, if $d: A \rightarrow A \otimes A$ is any derivation then the fact that $e$ is idempotent implies that $d(e) \in e A \otimes A f \oplus f A \otimes A e$. Thus there is an inner derivation $d^{\prime} \in I$ with $\left(d+d^{\prime}\right)(e)=0$. It follows that $D+I=\operatorname{Der}(A, A \otimes A)$.

Theorem 6.2. If $A=B \times C, \lambda \in K \otimes_{\mathbb{Z}} \mathrm{K}_{0}(B), \mu \in K \otimes_{\mathbb{Z}} \mathrm{K}_{0}(C)$, and $(\lambda, \mu)$ is the corresponding element of $K \otimes_{\mathbb{Z}} \mathrm{K}_{0}(A)$, then $\Pi^{(\lambda, \mu)}(A) \cong \Pi^{\lambda}(B) \times \Pi^{\mu}(C)$.

Proof. It suffices to prove that if $a \in A$ then $\Pi^{a}(A) \cong \Pi^{u}(B) \times \Pi^{v}(C)$ where $u=e a e$ and $v=f a f$. Now

$$
\begin{aligned}
\Pi^{a}(A) & =\mathrm{T}_{A} \operatorname{Der}(A, A \otimes A) /(\Delta-u-v) \\
& =\mathrm{T}_{A} \operatorname{Der}(A, A \otimes A) /(e \Delta f, f \Delta e, e \Delta e-u, f \Delta f-v) \\
& \cong \mathrm{T}_{A}[\operatorname{Der}(B, B \otimes B) \oplus \operatorname{Der}(C, C \otimes C)] /\left(\Delta_{e A e}-u, \Delta_{f A f}-v\right)
\end{aligned}
$$


by the previous lemma. Now this last tensor algebra is isomorphic to

$$
\mathrm{T}_{B} \operatorname{Der}(B, B \otimes B) \times \mathrm{T}_{C} \operatorname{Der}(C, C \otimes C),
$$

and the result follows.

\section{Bimodule-finite algebras}

Lemma 7.1. If $A \rightarrow B$ is a ring epimorphism then there is an exact sequence of $B$-B-bimodules $0 \rightarrow \operatorname{Tor}_{1}^{A}(B, B) \rightarrow B \otimes_{A} \Omega^{1} A \otimes_{A} B \rightarrow \Omega^{1} B \rightarrow 0$. Thus if $A$ is bimodule-finite then so is $B$.

Proof. Take the defining exact sequence for $\Omega^{1} A$ and tensor each side with $B$. Now use the fact that multiplication induces an isomorphism $B \otimes_{A} B \rightarrow B$.

There is the following obvious consequence.

Lemma 7.2. If $A \rightarrow B$ is a pseudoflat ring epimorphism then $\Omega^{1} B$ is isomorphic to $B \otimes_{A} \Omega^{1} A \otimes_{A} B$. Thus if $A$ is quasi-free then so is $B$.

Proposition 7.3. An algebra $A$ is bimodule-finite if and only if it has a finitely generated subalgebra $C$, such that the inclusion $C \rightarrow A$ is a ring epimorphism.

Proof. If there is such a subalgebra $C$ then $\Omega^{1} C$ is known to be finitely generated (see for example [1]), and the short exact sequence of Lemma 7.1 shows that $\Omega^{1} A$ is finitely generated. For the converse, choose generators of $\Omega^{1} A$, and choose a finitely generated subalgebra $C$ sufficiently large so that the generators all lie in $C \otimes C \subseteq A \otimes A$. Now there is a commutative diagram with exact rows

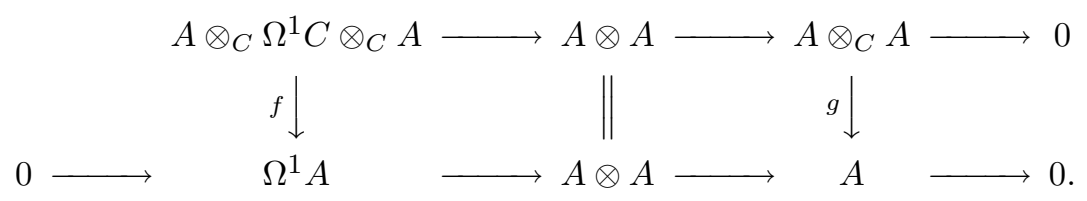

By construction $f$ is surjective, and it follows that $g$ is injective. In other words the inclusion $C \rightarrow A$ is a ring epimorphism. 


\section{Finite-dimensional quasi-free algebras}

Lemma 8.1. Let $M$ be an A-A-bimodule which is flat as a right A-module. If $M \otimes_{A} X$ is flat for all left $A$-modules $X$, then $\operatorname{Tor}_{1}^{A^{e}}(X \otimes Y, M)=0$ for all left $A$-modules $X$ and right $A$-modules $Y$.

Proof. Fix an exact sequence $0 \rightarrow L \rightarrow P \rightarrow M \rightarrow 0$ with $P$ a projective $A$ - $A$ bimodule. Tensoring first with $X$ and then with $Y$, the hypotheses imply that the tensor product sequence

$$
0 \rightarrow Y \otimes_{A} L \otimes_{A} X \rightarrow Y \otimes_{A} P \otimes_{A} X \rightarrow Y \otimes_{A} M \otimes_{A} X \rightarrow 0
$$

is exact. But this sequence is identified with the sequence

$$
0 \rightarrow(X \otimes Y) \otimes_{A^{e}} L \rightarrow(X \otimes Y) \otimes_{A^{e}} P \rightarrow(X \otimes Y) \otimes_{A^{e}} M \rightarrow 0
$$

so $\operatorname{Tor}_{1}^{A^{e}}(X \otimes Y, M)=0$.

Lemma 8.2. Suppose that $A$ is a finite-dimensional algebra, and that $A / \operatorname{rad} A$ is separable over $K$. Let $M$ be an $A$-A-bimodule which is projective as a right $A$ module. If $M \otimes_{A} X$ is projective for any left $A$-module $X$, then $M$ is a projective bimodule.

Proof. Since $\operatorname{rad} A$ is nilpotent, every simple $A$ - $A$-bimodule occurs as a composition factor of $(A / \operatorname{rad} A) \otimes(A / \operatorname{rad} A)$, and the separability hypothesis implies that $(A / \operatorname{rad} A) \otimes(A / \operatorname{rad} A)$ is semisimple. The previous lemma now shows that $\operatorname{Tor}_{1}^{A^{e}}(S, M)=0$ for all simple $A^{e}$-modules $S$. Since $A^{e}$ is finite-dimensional, it follows that $M$ is a projective $A^{e}$-module.

Lemma 8.3. If $A$ and $B$ are finite-dimensional self-injective algebras, then so is $A \otimes B$.

Proof. If $D$ is duality with the field, then $D(A)$ is a projective $A$-module and $D(B)$ is a projective $B$-module. Now the isomorphism $D(A \otimes B) \cong D(A) \otimes D(B)$ shows that $D(A \otimes B)$ is a projective $A \otimes B$-module, so $A \otimes B$ is self-injective.

Lemma 8.4. If $A$ is a finite-dimensional self-injective algebra then every $A$ module is either projective, or has infinite projective dimension.

Proof. Looking at the last two terms in the minimal projective resolution of a module of finite projective dimension, there must be an injection of one projective into another which is not split. This is impossible if all projective modules are injective. 
Proposition 8.5. A finite-dimensional algebra $A$ is quasi-free if and only if it is hereditary and $A / \operatorname{rad} A$ is separable over $K$.

Proof. If $A$ is hereditary, the bimodule $\Omega^{1} A$ satisfies the hypotheses of Lemma 8.2. Thus if $A / \operatorname{rad} A$ is separable over $K$ then $\Omega^{1} A$ is a projective bimodule, so $A$ is quasi-free.

Now suppose that $A$ is quasi-free. Certainly this implies that $A$ is hereditary. Let $S$ be a simple $A$-module, and let $B$ be the corresponding simple factor of $A / \operatorname{rad} A$. Since $A$ is hereditary, its Gabriel quiver has no oriented cycles (since any nonzero map between indecomposable projectives must be injective). It follows that $\operatorname{Ext}_{A}^{1}(S, S)=0$. Now the projection $A \rightarrow B$ is a pseudoflat epimorphism by [15, Theorem 4.8], since the the restriction to $A$ of any $B$-module is isomorphic to a direct sum of copies of $S$. Thus $B$ is quasi-free, so $B$ has projective dimension $\leq 1$ as a $B^{e}$-module. Now $B^{e}$ is self-injective by Lemma 8.3 , so actually $B$ is a projective module. Thus $B$ is separable. Repeating for each simple $A$-module it follows that $A / \operatorname{rad} A$ is separable.

\section{Localization}

Lemma 9.1. If $\theta: A \rightarrow B$ is a pseudoflat epimorphism and $M$ is a B-B-bimodule, then restriction induces an bijection $\operatorname{Der}(B, M) \rightarrow \operatorname{Der}(A, M)$.

Proof. We have isomorphisms

$$
\operatorname{Hom}_{B^{e}}\left(\Omega^{1} B, M\right) \cong \operatorname{Hom}_{B^{e}}\left(B \otimes_{A} \Omega^{1} A \otimes_{A} B, M\right) \cong \operatorname{Hom}_{A^{e}}\left(\Omega^{1} A, M\right)
$$

using Lemma 7.2.

Lemma 9.2. If $\theta: A \rightarrow B$ is a pseudoflat epimorphism and $\lambda \in K \otimes_{\mathbb{Z}} \mathrm{K}_{0}(A)$ then there is a natural homomorphism $\Pi^{\lambda}(A) \rightarrow \Pi^{\theta_{*}(\lambda)}(B)$. Moreover the diagram

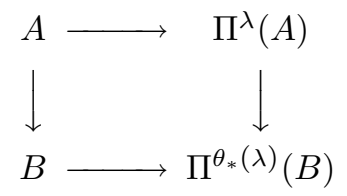

commutes.

Proof. The question of naturality is slightly delicate, since the definition of $\Pi^{\lambda}(A)$ depends on the choice of a lift of $\operatorname{tr}(\lambda)$ to $A$, and for the homomorphism from $\Pi^{\lambda}(A)$ to $\Pi^{\theta_{*}(\lambda)}(B)$ one should choose compatible lifts. The map $\theta$ induces a map 
$\bar{\theta}: A /[A, A] \rightarrow B /[B, B]$, and the square

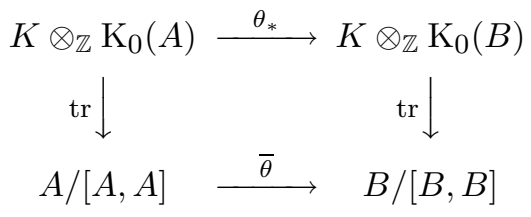

commutes. If $a$ is a lift to $A$ of $\operatorname{tr}(\lambda)$ we use $\theta(a)$ as a lift of $\theta_{*}(\lambda)$. Thus it suffices to construct a natural map $\Pi^{a}(A) \rightarrow \Pi^{\theta(a)}(B)$. Now there is a natural map

$$
\operatorname{Der}(A, A \otimes A) \rightarrow \operatorname{Der}(A, B \otimes B) \cong \operatorname{Der}(B, B \otimes B)
$$

which is in fact a homomorphism of $A$-A-bimodules, and it sends $\Delta_{A}$ to $\Delta_{B}$. Combining this with the algebra map $A \rightarrow B$ one obtains an algebra map

$$
\mathrm{T}_{A} \operatorname{Der}(A, A \otimes A) \rightarrow \mathrm{T}_{B} \operatorname{Der}(B, B \otimes B) .
$$

This map sends $\Delta_{A}$ to $\Delta_{B}$ and $a$ to $\theta(a)$. The result follows.

Theorem 9.3. Suppose that $\theta: A \rightarrow B$ is a pseudoflat epimorphism and that $\lambda \in K \otimes_{\mathbb{Z}} \mathrm{K}_{0}(A)$. If $A$ is a quasi-free bimodule-finite algebra, then so is $B$, and the diagram

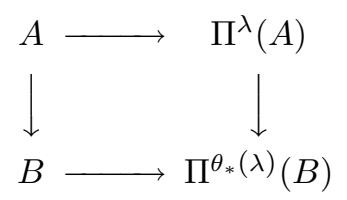

is a pushout in the category of rings.

Proof. We have $B \otimes_{A} \operatorname{Hom}_{A^{e}}\left(\Omega^{1} A, A \otimes A\right) \otimes_{A} B \cong \operatorname{Hom}_{A^{e}}\left(\Omega^{1} A, B \otimes B\right)$ since $\Omega^{1} A$ is a finitely generated projective $A$ - $A$-bimodule. Thus the induced map

$$
B \otimes_{A} \operatorname{Der}(A, A \otimes A) \otimes_{A} B \rightarrow \operatorname{Der}(B, B \otimes B)
$$

is an isomorphism of $B$ - $B$-bimodules, sending $1 \otimes \Delta_{A} \otimes 1$ to $\Delta_{B}$. Let $a$ be a lift of $\operatorname{tr}(\lambda)$ to $A$. Since $\Pi^{a}(A) \cong\left(\mathrm{T}_{A} \operatorname{Der}(A, A \otimes A)\right) /\left(\Delta_{A}-a\right)$, the pushout of $A \rightarrow B$ and $A \rightarrow \Pi^{a}(A)$ is isomorphic to

$$
\mathrm{T}_{B}\left(B \otimes_{A} \operatorname{Der}(A, A \otimes A) \otimes_{A} B\right) /\left(1 \otimes \Delta_{A} \otimes 1-\theta(a)\right),
$$

and this is isomorphic to $\Pi^{\theta_{*}(\lambda)}(B)$.

Corollary 9.4. Under the hypotheses of the theorem, the map $\Pi^{\lambda}(A) \rightarrow \Pi^{\theta_{*}(\lambda)}(B)$ is a pseudoflat epimorphism. 


\section{Proof. Use [1, Proposition 5.2].}

We shall also need one further observation.

Corollary 9.5. Under the hypotheses of the theorem, the $\operatorname{map} \Pi^{\lambda}(A) \rightarrow \Pi^{\theta_{*}(\lambda)}(B)$ preserves filtrations, that is it sends $\Pi^{\lambda}(A)_{\leq n}$ into $\Pi^{\theta_{*}(\lambda)}(B)_{\leq n}$ for all $n$. Moreover, it induces a commutative square

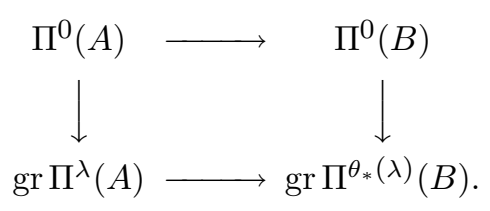

One of the main examples of a pseudoflat epimorphism is given by universal localization, see [1]. Here we consider just the special case arising from perpendicular categories. If $A$ is hereditary and $\mathcal{X}$ is a collection of finitely presented left $A$-modules, then the perpendicular category $\mathcal{X}^{\perp}$ is the category of modules $M$ with $\operatorname{Hom}_{A}(X, M)=\operatorname{Ext}^{1}(X, M)=0$ for all $X \in \mathcal{X}$. Considering the universal localization of $A$ with respect to projective presentations of the modules in $X$, one obtains a pseudoflat epimorphism $A \rightarrow A_{\mathcal{X}}$ with the property that restriction induces an equivalence between the category of left $A_{\mathcal{X}}$-modules and $\mathcal{X}^{\perp}$. The theorem now implies the following.

Corollary 9.6. Suppose that $A$ is quasi-free and bimodule-finite and $\lambda \in K \otimes_{\mathbb{Z}}$ $\mathrm{K}_{0}(A)$. If $\mathcal{X}$ is a collection of finitely presented left $A$-modules and $\theta: A \rightarrow A_{\mathcal{X}}$ is the corresponding universal localization, then restriction via the natural map induces an equivalence from the category of $\Pi^{\theta_{*}}(\lambda)\left(A_{\mathcal{X}}\right)$-modules to the category of $\Pi^{\lambda}(A)$-modules whose restriction to $A$ is in $\mathcal{X}^{\perp}$.

\section{Module varieties}

Let $K$ be an algebraically closed field, let $A$ be a finitely generated $K$-algebra and let $e_{i}(i \in I)$ be a complete set of orthogonal idempotents, so $e_{i} e_{j}=0$ for $i \neq j$ and $\sum_{i} e_{i}=1$. If $\alpha \in \mathbb{N}^{I}$, we write $\operatorname{Rep}(A, \alpha)$ for the variety of $A$-module structures on $K^{\alpha}=\bigoplus_{i} K^{\alpha_{i}}$ under which each $e_{i}$ acts as projection onto the $i$ th summand. Thus $\operatorname{Rep}(A, \alpha)$ consists of all algebra maps $A \rightarrow \operatorname{End}_{K}\left(K^{\alpha}\right)$ sending $e_{i}$ to the projection onto $K^{\alpha_{i}}$. Elements of $\operatorname{Rep}(A, \alpha)$ are representations of $A$ of dimension vector $\alpha$. The group $\operatorname{GL}(\alpha)=\prod_{i} \operatorname{GL}\left(\alpha_{i}\right)$ acts naturally on this variety, and the orbits correspond to isomorphism classes of representations. The stabilizer of a representation $X$ can be identified with the automorphism group of $X$. It follows that the orbit of $X$ has dimension $\sum_{i} \alpha_{i}^{2}-\operatorname{dim} \operatorname{End}(X)$. 
Lemma 10.1. If $\beta \leq \alpha$ then the set of elements of $\operatorname{Rep}(A, \alpha)$ such that the corresponding representation has a subrepresentation of dimension vector $\beta$, is closed.

Proof. It is the image of a closed set under the projection $\operatorname{Rep}(A, \alpha) \times P \rightarrow$ $\operatorname{Rep}(A, \alpha)$, where $P$ is the product of Grassmannians of subspaces of dimension $\beta_{i}$ in $K^{\alpha_{i}}$. Now use the fact that $P$ is a projective variety. See [16, Lemma 3.1].

We write $\operatorname{Rep}(A, \alpha)_{s}$ for the $\operatorname{GL}(\alpha)$-stable subset consisting of simple $A$-modules. By the lemma it is open.

Lemma 10.2. If $A$ is a finitely generated $K$-algebra of $G K$ dimension $d$, then for any $\alpha$ we have $\operatorname{dim} \operatorname{Rep}(A, \alpha)_{s} \leq \sum_{i} \alpha_{i}^{2}+d-1$.

Proof. Passing to the quotient of $A$ by the intersection of the annihilators of all simple representations of dimension vector $\alpha$, we may suppose that $A$ is a semiprime PI ring, satisfying the identities of $N \times N$ matrices, where $N=\sum_{i} \alpha_{i}$. By [11, Theorem 13.4.4], $A$ has only finitely many minimal prime ideals, so we may assume that it is prime. By [11, Proposition 13.7.4] there is a central localization $A_{c}$ which is an Azumaya algebra. Now each simple $A$-module is either an $A_{c}$-module, or an $A /(c)$-module, so by an induction we reduce to the case when $A$ is an Azumaya algebra, say with centre $Z$.

Now there is a natural map $f: \operatorname{Rep}(A, \alpha)_{s} \rightarrow$ maxspec $Z$ sending a simple $A$ module to its central character. For, $Z$ is finitely generated by [11, Lemma 13.9.10], and if $z_{1}, \ldots, z_{r}$ are generators, they identify maxspec $Z$ with a closed subset of $K^{r}$. Now if $N=\sum_{i} \alpha_{i}$ then $\operatorname{End}_{K}\left(K^{\alpha}\right) \cong \mathrm{M}_{N}(K)$, and if $\theta: A \rightarrow \mathrm{M}_{N}(K)$ is an element of $\operatorname{Rep}(A, \alpha)_{s}$ then the map $z \mapsto \theta(z)_{11}$ is the central character of $\theta$. Thus we can define $f$ by sending $\theta$ to $\left(\theta\left(z_{1}\right)_{11}, \ldots, \theta\left(z_{r}\right)_{11}\right) \in K^{r}$.

Now each fibre of $f$ meets only finitely many $\operatorname{GL}(\alpha)$-orbits, and each orbit has dimension $\sum_{i} \alpha_{i}^{2}-1$. The result follows since $Z$ has Krull dimension at most $d$.

Now let $Q$ be a quiver with vertex set $I$ and let $\alpha \in \mathbb{N}^{I}$. Clearly $\operatorname{Rep}(K Q, \alpha)$ can be identified with the affine space

$$
\bigoplus_{a: i \rightarrow j} \operatorname{Hom}_{K}\left(K^{\alpha_{i}}, K^{\alpha_{j}}\right)
$$

Also, by Section 3 and [5], one can identify $\operatorname{Rep}\left(\Pi^{0}(K Q), \alpha\right)$ with the fibre over 0 of the moment map

$$
\mu: \operatorname{Rep}(K \bar{Q}, \alpha) \rightarrow \operatorname{End}(\alpha)_{0}, \quad x \mapsto \sum_{a \in Q}\left[x_{a}, x_{a^{*}}\right],
$$

where $\operatorname{End}(\alpha)=\prod_{i} \operatorname{End}\left(K^{\alpha_{i}}\right)$ and $\operatorname{End}(\alpha)_{0}=\left\{\left(\theta_{i}\right) \in \operatorname{End}(\alpha) \mid \sum_{i} \operatorname{tr}\left(\theta_{i}\right)=0\right\}$. Recall that a module $M$ is called a brick if $\operatorname{End}(M)=K$. 
Lemma 10.3. The map $\mu$ is smooth at a point $x \in \operatorname{Rep}(K \bar{Q}, \alpha)$ if and only if the corresponding module is a brick.

Proof. Identifying $\operatorname{Rep}(K \bar{Q}, \alpha)$ and $\operatorname{End}(\alpha)_{0}$ with their tangent spaces at $x$ and $\mu(x)$ respectively, $\mu$ induces the map

$$
\mathrm{d} \mu_{x}: \operatorname{Rep}(K \bar{Q}, \alpha) \rightarrow \operatorname{End}(\alpha)_{0}, \quad y \mapsto \sum_{a \in Q}\left(\left[x_{a}, y_{a^{*}}\right]+\left[y_{a}, x_{a^{*}}\right]\right) .
$$

Now if $D$ is duality with the field, there is a trace pairing which identifies the vector spaces $D(\operatorname{Rep}(K \bar{Q}, \alpha)) \cong \operatorname{Rep}(K \bar{Q}, \alpha)$ (with arrows $a$ and $a^{*}$ being interchanged), and identifies $D\left(\operatorname{End}(\alpha)_{0}\right) \cong \operatorname{End}(\alpha) / K$. Then $D\left(\mathrm{~d} \mu_{x}\right)$ is the map

$$
\begin{gathered}
\operatorname{End}(\alpha) / K \rightarrow \operatorname{Rep}(K \bar{Q}, \alpha), \\
\left(\theta_{i}\right) \mapsto \sum_{a: i \rightarrow j \in Q}\left(\left(\theta_{j} x_{a}-x_{a} \theta_{i}\right)_{a^{*}}+\left(x_{a^{*}} \theta_{j}-\theta_{i} x_{a^{*}}\right)_{a}\right),
\end{gathered}
$$

so if $X$ is the $\Pi^{0}(K Q)$-module corresponding to $x$, then $\operatorname{Ker} D\left(\mathrm{~d} \mu_{x}\right) \cong \operatorname{End}(X) / K$. Now $X$ is a brick if and only if $D\left(\mathrm{~d} \mu_{x}\right)$ is injective, so if and only if $\mu$ is smooth at $x$.

Now let $Q$ be an extended Dynkin quiver and let $\delta$ be the minimal positive imaginary root for $Q$.

Lemma 10.4. The restriction map $\pi: \operatorname{Rep}\left(\Pi^{0}(K Q), \delta\right) \rightarrow \operatorname{Rep}(K Q, \delta)$ is onto, and the fibre over a point $x \in \operatorname{Rep}(K Q, \delta)$ is irreducible of dimension $\operatorname{dim} \operatorname{End}(X)$, where $X$ is the $K Q$-module corresponding $x$.

Proof. The fibre over $x$ is isomorphic to the fibre $c^{-1}(0)$ in [5, Lemma 4.2], so it is isomorphic to $D \operatorname{Ext}^{1}(X, X)$. Now since $X$ has dimension $\delta$ the Ringel form implies that this has dimension $\operatorname{dim} \operatorname{End}(X)$.

Lemma 10.5. The variety $\operatorname{Rep}\left(\Pi^{0}(K Q), \delta\right)$ is irreducible and Cohen-Macaulay of dimension $1+\sum_{i} \delta_{i}^{2}$, and the general element is a simple $\Pi^{0}(K Q)$-module. Moreover, if $\operatorname{Rep}\left(\Pi^{0}(K Q), \delta\right)$ is considered as a scheme using the natural scheme structure on the fibre $\mu^{-1}(0)$, then it is reduced.

Proof. Equip $\operatorname{Rep}\left(\Pi^{0}(K Q), \delta\right)$ with the scheme structure. The argument of $[5$, Lemma 8.3] shows that $\operatorname{Rep}\left(\Pi^{0}(K Q), \delta\right)$ is irreducible of dimension $1+\sum_{i} \delta_{i}^{2}$, hence a complete intersection, so Cohen-Macaulay.

Supposing that the general element is not simple, it follows from the irreducibility and Lemma 10.1 that there is $0<\beta<\delta$ such that every $\Pi^{0}(K Q)$-module of dimension $\delta$ has a subrepresentation of dimension $\beta$. Now any $K Q$-module of 
dimension $\delta$ can be considered trivially as a $\Pi^{0}(K Q)$-module, so has a submodule of dimension $\beta$. Similarly any $K Q^{o p}$-module of dimension $\delta$ can be considered as a $\Pi^{0}(K Q)$-module, so has a submodule of dimension $\beta$. Dualizing, this implies that any $K Q$-module of dimension $\delta$ has a submodule of dimension $\delta-\beta$. This is impossible by [16, Theorem 3.4].

Now $\mu$ is smooth at the general point of $\operatorname{Rep}\left(\Pi^{0}(K Q), \delta\right)$ by Lemma 10.3, so $\operatorname{Rep}\left(\Pi^{0}(K Q), \delta\right)$ is generically smooth, hence generically reduced. With the Cohen-Macaulay property, this implies that it is reduced.

\section{A Conze embedding}

Let $Q$ be an extended Dynkin quiver with vertex set $I$ and let $\delta \in \mathbb{Z}^{I}$ be the minimal positive imaginary root for $Q$. Let $K$ be an algebraically closed field.

Lemma 11.1. There is a pseudoflat epimorphism $\theta: K Q \rightarrow \mathrm{M}_{N}(K[x])$ such that the general representation of $K Q$ of dimension $\delta$ is the restriction of a $\mathrm{M}_{N}(K[x])$ module.

Proof. If $Q$ is of type $\tilde{A}_{n}$, oriented as a cycle, then $\mathcal{X}$ should consist of $n$ of the $n+1$ one-dimensional simple modules. If $Q$ has no oriented cycles then $\mathcal{X}$ should contain all the regular simple modules in one tube in the Auslander-Reiten quiver of $K Q$, and all but one regular simple module in every other tube. Localizing at a set which contains all but one regular simple in each tube, one obtains by $[4$, Theorem 4.2] a tame hereditary algebra with two simple modules. Since the base field is algebraically closed, this algebra is Morita equivalent to the Kronecker algebra. Now localizing at one further regular simple module, one obtains an algebra Morita equivalent to $K[x]$.

Henceforth we suppose that $\theta: K Q \rightarrow \mathrm{M}_{N}(K[x])$ is a pseudoflat epimorphism such that the general representation of $K Q$ of dimension $\delta$ is the restriction of a $\mathrm{M}_{N}(K[x])$-module. If $\lambda \in K \otimes_{\mathbb{Z}} \mathrm{K}_{0}(K Q)$ there is a corresponding element $\boldsymbol{\lambda} \in K^{I}$. See Section 3.

Lemma 11.2. We have $N=\sum_{i} \delta_{i}$, and if $P$ is the indecomposable projective module for $\mathrm{M}_{N}(K[x])$, then $\theta_{*}(\lambda)=\sum_{i} \boldsymbol{\lambda}_{i} \delta_{i} \otimes[P]$ for $\lambda \in K \otimes_{\mathbb{Z}} \mathrm{K}_{0}(K Q)$.

Proof. The general representation of $K Q$ of dimension $\delta$ is a brick, so it must be the restriction of a simple $\mathrm{M}_{N}(K[x])$-module. The result follows.

Recall from the introduction that $\theta$ induces a map $\theta_{\lambda}$ from $\Pi^{\lambda}(K Q)$ to $\mathrm{M}_{N}\left(C_{\nu}\right)$ for some $\nu$. The previous lemma and Corollary 5.4 show that $\nu=\sum_{i} \boldsymbol{\lambda}_{i} \delta_{i}$. 
Lemma 11.3. The map $\theta_{0}$ is injective.

Proof. We factorize $\theta_{0}$ as a product

$$
\Pi^{0}(K Q) \stackrel{f}{\rightarrow} \Pi^{0}(K Q) / \operatorname{Ker}\left(\theta_{0}\right) \stackrel{g}{\rightarrow} \mathrm{M}_{N}\left(C_{0}\right) .
$$

Both of these maps are ring epimorphisms, so we obtain injective morphisms

$$
\operatorname{Rep}\left(\mathrm{M}_{N}\left(C_{\nu}\right), \delta\right) \rightarrow \operatorname{Rep}\left(\Pi^{0}(K Q) / \operatorname{Ker}\left(\theta_{0}\right), \delta\right) \rightarrow \operatorname{Rep}\left(\Pi^{0}(K Q), \delta\right) .
$$

The image of the composition of these is the open set of representations whose restriction to $K Q$ is in $\mathcal{X}^{\perp}$. Now Lemma 10.5 implies that $\operatorname{Rep}\left(\Pi^{0}(K Q) / \operatorname{Ker}\left(\theta_{0}\right), \delta\right)_{s}$ has dimension $1+\sum_{i} \delta_{i}^{2}$. Thus $\Pi^{0}(K Q) / \operatorname{Ker}\left(\theta_{0}\right)$ has GK dimension at least 2 by Lemma 10.2. Now $\Pi^{0}(K Q)$ is prime of GK dimension 2 by [2], and it follows that $\operatorname{Ker}\left(\theta_{0}\right)=0$, as claimed.

Theorem 11.4. The map $\theta_{\lambda}$ is injective, the natural map $\Pi^{0}(K Q) \rightarrow \operatorname{gr} \Pi^{\lambda}(K Q)$ is an isomorphism, and $\Pi^{\lambda}(K Q)$ is prime of GK dimension 2.

Proof. By Corollary 9.5 there is a commutative square

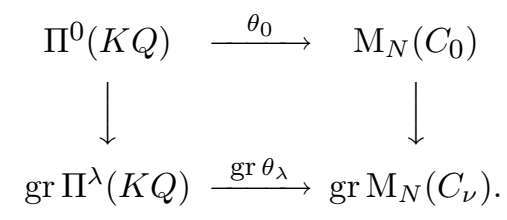

The associated graded algebra for the first Weyl algebra is the polynomial ring in two variables, so the map $\mathrm{M}_{N}\left(C_{0}\right) \rightarrow \operatorname{gr~} \mathrm{M}_{N}\left(C_{\nu}\right)$ is an isomorphism. Now the map $\Pi^{0}(K Q) \rightarrow \operatorname{gr} \Pi^{\lambda}(K Q)$ is surjective, so the fact that $\theta_{0}$ is injective implies that $\Pi^{0}(K Q) \rightarrow \operatorname{gr} \Pi^{\lambda}(K Q)$ is an isomorphism and $\operatorname{gr} \theta_{\lambda}$ is injective. It follows that $\theta_{\lambda}$ is injective. Finally, $\Pi^{\lambda}(K Q)$ is prime of GK dimension 2 by [2].

Lemma 11.5. The ring $\Pi^{\lambda}(K Q)$ has Goldie rank at least $N$.

Proof. Let $M$ be the restriction to $\Pi^{\lambda}(K Q)$ of the simple module for its quotient ring. Letting $E=\operatorname{End}(M)^{o p}$ we consider $M$ as a $\Pi^{\lambda}(K Q)$ - $E$-bimodule. One can consider $M$ as a representation of the quiver $\bar{Q}$ by right $E$-vector spaces and $E$ linear maps, satisfying the usual relations for the deformed preprojective algebra. Now these vector spaces are finite dimensional over $E$, and lead to a dimension vector $\alpha \in \mathbb{N}^{I}$, and the Goldie rank of $\Pi^{\lambda}(K Q)$ is $\sum_{i} \alpha_{i}$.

If $i$ is a loopfree vertex and $\lambda_{i} \neq 0$ then the reflection functor of [5, Theorem $5.1]$ evidently defines an equivalence from the category of $\Pi^{\lambda}(K Q)$ - $E$-bimodules to the category of $\Pi^{\lambda^{\prime}}(K Q)$-E-bimodules, for some $\lambda^{\prime}$, which acts as $s_{i}$ on dimension vectors. 
By applying a sequence of reflection functors to $M$ we pass to a $\Pi^{\lambda^{\prime}}(K Q)-E$ bimodule $M^{\prime}$ of dimension vector $\alpha^{\prime}$ (for a new $\lambda^{\prime}$ ), and we choose the sequence to ensure that $\alpha^{\prime}$ is minimal. This implies that for any vertex $i$, either $\lambda_{i}^{\prime}=0$ or $\left(\alpha^{\prime}, \epsilon_{i}\right) \leq 0$.

If $i$ is a vertex with $\lambda_{i}^{\prime}=0$, the 1 -dimensional simple module $S_{i}$ at vertex $i$ has projective resolution

$$
0 \rightarrow \Pi^{\lambda}(K Q) e_{i} \rightarrow \bigoplus_{a: i \rightarrow j \text { in } \bar{Q}} \Pi^{\lambda}(K Q) e_{j} \rightarrow \Pi^{\lambda}(K Q) e_{i} \rightarrow S_{i} \rightarrow 0 .
$$

Now $M$ is an injective module over $\Pi^{\lambda}(K Q)$, so $M^{\prime}$ is injective over $\Pi^{\lambda^{\prime}}(K Q)$, so applying $\operatorname{dim}_{E} \operatorname{Hom}\left(-, M^{\prime}\right)$ we deduce that $\left(\alpha^{\prime}, \epsilon_{i}\right)=0$.

Thus $\alpha^{\prime}$ is in the fundamental region for $Q$, so is a multiple of the vector $\delta$. Now $\delta$ is unchanged by the reflections $s_{i}$, so $\alpha$ must have been a multiple of $\delta$. The result follows.

Let $D_{\nu}$ be the quotient division algebra for $C_{\nu}$.

Theorem 11.6. The map $\theta_{\lambda}$ induces an isomorphism from the simple artinian quotient ring of $\Pi^{\lambda}(K Q)$ to $\mathrm{M}_{N}\left(D_{\nu}\right)$.

Proof. First observe that if $k<N$ and $D$ and $E$ are division rings, then there can be no homomorphism $\mathrm{M}_{N}(E) \rightarrow \mathrm{M}_{k}(D)$, for if $S$ is the module obtained by inducing the simple $\mathrm{M}_{N}(E)$-module up to $\mathrm{M}_{k}(D)$, then $S^{N} \cong \mathrm{M}_{k}(D)$ is semisimple of length $k$.

By [11, Proposition 3.1.16], the quotient ring of $\Pi^{\lambda}(K Q)$ embeds in $\mathrm{M}_{k}\left(D_{\nu}\right)$ for some $k \leq N$. By the discussion above we must have $k=N$, and inspecting the proof of the cited result we see that $\mathrm{M}_{N}\left(D_{\nu}\right)$ is simple as a $\Pi^{\lambda}(K Q)-\mathrm{M}_{N}\left(D_{\nu}\right)$ bimodule, and then that it is torsion free over $\Pi^{\lambda}(K Q)$. This means that $\theta_{\lambda}$ does induce a map from the quotient ring of $\Pi^{\lambda}(K Q)$ to $\mathrm{M}_{N}\left(D_{\nu}\right)$. Moreover this map is an isomorphism since it is a ring epimorphism.

We now apply this to Kleinian singularities. Let $K$ be an algebraically closed field of characteristic zero, and let $\Gamma$ be a finite subgroup of $\mathrm{SL}_{2}(K)$. Let $Q$ be an orientation of the McKay quiver of $\Gamma$. Recall that there is an isomorphism $\mathcal{O}^{\lambda} \cong e_{0} \Pi^{\lambda}(K Q) e_{0}$, where $\lambda \in Z(K \Gamma)$ is identified with $\boldsymbol{\lambda} \in K^{I}$ by letting $\lambda_{i}$ be the trace of $\lambda$ on the $i$ th irreducible representation of $\Gamma$, and hence with $\lambda \in K \otimes_{\mathbb{Z}} \mathrm{K}_{0}(K Q)$.

Corollary 11.7. There is an embedding $\psi_{\lambda}: \mathcal{O}^{\lambda} \rightarrow C_{\nu}$ where $\nu$ is the trace of $\lambda$ on the regular representation of $\Gamma$. Moreover $\psi_{\lambda}$ induces an isomorphism on quotient division rings.

Proof. It suffices to observe that if $e$ is an idempotent in a prime Goldie ring $R$ 
with simple artinian quotient $\operatorname{ring} Q(R)$, then $e$ Re is prime Goldie with quotient ring $e Q(R) e$.

\section{Appendix}

In this appendix we use the methods of the paper to prove the normality of a certain variety. This result is used in the article [9] by M. P. Holland. If $K$ is an algebraically closed field and $Q$ is an extended Dynkin quiver with minimal imaginary root $\delta$, then the variety $\operatorname{Rep}\left(\Pi^{0}(K Q), \delta\right)$ need not be normal (see below). Here we prove the normality of the open subvariety $\operatorname{Rep}\left(\Pi^{0}(K Q), \delta\right)_{s s}$ of semistable $\Pi^{0}(K Q)$-modules, where the semistable modules are defined as follows. If $M$ is a $K Q$ - or a $\Pi^{0}(K Q)$-module of dimension vector $\alpha$, then its defect is defined by the formula $d(M)=\langle\delta, \alpha\rangle$. One says that $M$ is semistable if $d(M)=0$ and $d(N) \leq 0$ for all submodules $N \subseteq M$. It is well known that the semistable $K Q$-modules are exactly the regular modules.

Lemma 12.1. $A \Pi^{0}(K Q)$-module is semistable if and only if it is semistable as a KQ-module.

Proof. Certainly if $M$ is semistable as a $K Q$-module then it is as a $\Pi^{0}(K Q)$-module, for any $\Pi^{0}(K Q)$-submodule $N$ is also a $K Q$-submodule, so $d(N) \leq 0$.

Now suppose that $M$ is semistable as a $\Pi^{0}(K Q)$-module. To show that it is semistable over $K Q$, it suffices to show that all indecomposable $K Q$-submodules of $M$ are preprojective or regular. For a contradiction, let $N$ be an indecomposable preinjective submodule. Now by Theorem 2.3,

$$
\Pi^{0}(K Q) \otimes_{K Q} N \cong N \oplus \tau^{-} N \oplus \tau^{-2}(N) \oplus \ldots,
$$

and since $N$ is preinjective this sum terminates, so it is a finite-dimensional preinjective $K Q$-module. Now the $\Pi^{0}(K Q)$-submodule $\bar{N}$ of $M$ generated by $N$ is a quotient of this sum, so it is preinjective as a $K Q$-module. Thus $d(\bar{N})>0$, contrary to the assumption.

Lemma 12.2. If $Q$ is an extended Dynkin quiver then any semistable $K Q$-module $M$ of dimension $\delta$ can be extended to a $\Pi^{0}(K Q)$-module which is a brick.

Proof. Certainly this is true if $Q$ has type $\tilde{A}_{n}$, so that $\delta_{i}=1$ for all vertices $i$. One considers the $K Q$-module $M$ as a representation of $Q$ in which the vector space at each vertex is 1-dimensional. Now one extends this to a representation of $\bar{Q}$ by letting $a^{*}$ be a non-zero map if and only if $a$ is zero. Clearly this defines a $\Pi^{0}(K Q)$-module, and it is easy to see that it is a brick.

To deal with other quivers, we first formulate the assertion in a Morita-invariant way, and then we use universal localization to reduce to type $\tilde{A}_{n}$. 
Observe that a $K Q$-module $M$ is semistable of dimension $\delta$ if and only if it is regular, and its regular composition factors are exactly the regular simples for some tube in the Auslander-Reiten quiver of $K Q$, each with multiplicity one. For simplicity we call this property $(*)$.

In view of the Morita equivalence property for $\Pi^{0}(A)$, the lemma may be formulated as the following claim: if $A$ is a tame hereditary algebra and $M$ is an $A$-module with property $\left({ }^{*}\right)$, then $M$ can be extended to a $\Pi^{0}(A)$-module which is a brick. We prove this claim by induction on the number of simple modules for $A$. Let $M$ be a module with property (*).

If there are no inhomogeneous tubes in the Auslander-Reiten quiver for $A$, except possibly the one containing $M$, then $A$ is of type $\tilde{A}_{n}$, and we have checked the claim at the start of the proof.

If there is such an inhomogeneous tube, choose a regular simple module $X$ contained in this tube, and let $A \rightarrow B$ be the corresponding universal localization. Then $B$ is a tame hereditary algebra with one fewer simple module than $A$ by [4, Theorem 4.2]. Now $M$ is in the perpendicular category to $X$, so it is the restriction of a $B$-module $M^{\prime}$, and clearly $M^{\prime}$ has property (*). By induction $M^{\prime}$ can be extended to a $\Pi^{0}(B)$-module $M^{\prime \prime}$ which is a brick, and then since $\Pi^{0}(A) \rightarrow \Pi^{0}(B)$ is a ring epimorphism, the restriction of $M^{\prime \prime}$ to $\Pi^{0}(A)$ is a brick. The claim follows.

Theorem 12.3. If $Q$ is an extended Dynkin quiver then $\operatorname{Rep}\left(\Pi^{0}(K Q), \delta\right)_{\text {ss }}$ is a normal variety.

Proof. It is Cohen-Macaulay by Lemma 10.5, so it suffices to prove that its singular locus $S$ has dimension at most $-1+\sum_{i} \delta_{i}^{2}$. Consider the projection

$$
\pi: \operatorname{Rep}\left(\Pi^{0}(K Q), \delta\right)_{s s} \rightarrow \operatorname{Rep}(K Q, \delta)_{s s} .
$$

Now the general element $r$ of $\operatorname{Rep}(K Q, \delta)_{s s}$ is a brick, so every element $x$ of the fibre $\pi^{-1}(r)$ is a brick. Thus by Lemma 10.3 the map $\mu$ is smooth at $x$, so $\operatorname{Rep}\left(\Pi^{0}(K Q), \delta\right)_{s s}$ is smooth at $x$. Besides the bricks, there are only finitely many $\operatorname{GL}(\delta)$-orbits of non-bricks in $\operatorname{Rep}(K Q, \delta)_{s s}$. If $\mathcal{O}_{X}$ is one of these orbits, then

$$
\operatorname{dim} \pi^{-1}\left(\mathcal{O}_{X}\right)=\operatorname{dim} \operatorname{End}(X)+\operatorname{dim} \mathcal{O}_{X}=\operatorname{dim} \operatorname{GL}(\delta)=\sum_{i} \delta_{i}^{2} .
$$

Now the general element of $\pi^{-1}\left(\mathcal{O}_{X}\right)$ is a brick by the previous lemma, and so $\pi^{-1}\left(\mathcal{O}_{X}\right) \cap S$ has dimension at most $-1+\sum_{i} \delta_{i}^{2}$, as required.

Finally we justify our claim that $\operatorname{Rep}\left(\Pi^{0}(K Q), \delta\right)$ need not be normal. By Lemma 10.5 the natural scheme structure on $\operatorname{Rep}\left(\Pi^{0}(K Q), \delta\right)$ is reduced, and hence in the notation of Section 10, the tangent space at a point $x \in \operatorname{Rep}\left(\Pi^{0}(K Q), \delta\right)$ can be identified with $\operatorname{Ker}\left(d \mu_{x}\right)$. It follows that $\operatorname{Rep}\left(\Pi^{0}(K Q), \delta\right)$ is smooth at $x$ if and only if $x$ is a brick. 
Now Ringel [12] has pointed out that if $Q$ is extended Dynkin, not of type $\tilde{A}_{n}$, then there is a $K Q$-module $X$ of dimension $\delta$ which is not the restriction of any brick for $\Pi^{0}(K Q)$. For example, let $I$ be an indecomposable injective $K Q$ module of defect $\geq 2$, say of dimension vector $\alpha$. Then $\delta-\alpha$ is a positive root, so is the dimension vector of an indecomposable $P$, necessarily preprojective. Let $X=P \oplus I$. The condition on defect implies that $\operatorname{Hom}(X, I)$ has dimension at least 2, so $\operatorname{Hom}(P, I) \neq 0$, and hence $X$ has a non-zero endomorphism $\phi$ which kills $I$ and has image contained in $I$. By Lemmas 1.4 and 2.2, a $\Pi^{0}(K Q)$-module structure on $X$ is determined by a map $\tau^{-} X \rightarrow X$. Now since $\tau^{-} I=0$ and $\operatorname{Hom}\left(\tau^{-} P, P\right)=0$, it is easy to see that $\phi$ is a $\Pi^{0}(K Q)$-endomorphism.

Now the inverse image of the $\mathrm{GL}(\delta)$-orbit of $X$ under the projection

$$
\operatorname{Rep}\left(\Pi^{0}(K Q), \delta\right) \rightarrow \operatorname{Rep}(K Q, \delta)
$$

has codimension 1 by the argument of Theorem 12.3. Since all points of the inverse image are singular, $\operatorname{Rep}\left(\Pi^{0}(K Q), \delta\right)$ cannot be normal.

\section{References}

[1] G. M. Bergman and W. Dicks, Universal derivations and universal ring constructions, $P a$ cific J. Math. 79 (1978), 293-337.

[2] D. Baer, W. Geigle and H. Lenzing, The preprojective algebra of a tame hereditary algebra, Commun. Algebra 15 (1987), 425-457.

[3] N. Conze, Algèbres d'opérateurs différentiels et quotients des algèbres enveloppantes, Bull. Soc. math. France 102 (1974), 379-415.

[4] W. Crawley-Boevey, Regular modules for tame hereditary algebras, Proc. London Math. Soc. 62 (1991), 490-508.

[5] W. Crawley-Boevey and M. P. Holland, Noncommutative deformations of Kleinian singularities, Duke Math. J. 92 (1998), 605-635.

[6] J. Cuntz and D. Quillen, Algebra extensions and nonsingularity, J. Amer. Math. Soc. 8 (1995), 251-289.

[7] V. Dlab and C. M. Ringel, The preprojective algebra of a modulated graph. In: Representation theory II, Proc. Ottawa 1979, eds V. Dlab and P. Gabriel, Lec. Notes in Math. 832, Springer, Berlin 1980, pp. 216-231.

[8] I. M. Gelfand and V. A. Ponomarev, Model algebras and representations of graphs, Funkcional Anal. i Priložen 13 (1979), 1-12. English translation: Func. Anal. Appl. 13 (1979), 157166.

[9] M. P. Holland, Quantization of the Marsden-Weinstein reduction for extended Dynkin quivers, to appear in Ann. Sci. École Norm. Sup.

[10] H. Matsumura, Commutative Ring Theory, Cambridge Univ. Press 1986.

[11] J. C. McConnell and J. C. Robson, Noncommutative noetherian rings, Wiley 1987.

[12] C. M. Ringel, private communication, 30 July 1997.

[13] C. M. Ringel, The preprojective algebra of a quiver, in: Algebras and modules II, Proc. Geiranger 1996, Canad. Math. Soc. Conf. Proc. 24, Amer. Math. Soc. 1998, 467-480.

[14] W. F. Schelter, Smooth Algebras, J. Algebra 103 (1986), 677-685.

[15] A. Schofield, Representations of rings over skew fields, London Math. Soc. Lec. Note Ser. 92, Cambridge Univ. Press 1985.

[16] A. Schofield, General representations of quivers, Proc. London Math. Soc. 65 (1992), 46-64. 
William Crawley-Boevey

Department of Pure Mathematics

University of Leeds

Leeds LS2 9JT

UK

e-mail: w.crawley-boevey@leeds.ac.uk

(Received: March 4, 1998) 